\title{
LA ACREDITACIÓN DE LA CALIDAD EDUCATIVA Y LA PERCEPCIÓN DE SU IMPACTO EN LA GESTIÓN ACADÉMICA: EL CASO DE UNA INSTITUCIÓN DEL SECTOR NO UNIVERSITARIO EN MÉXICO
}

\author{
Iván Alejandro Salas Durazo ${ }^{1}$
}

\begin{abstract}
RESUMEN
El presente artículo analiza la acreditación de los programas educativos y la percepción de su impacto en la gestión institucional. Para llevar esto a cabo se consideraron como ejes de análisis la calidad del sistema educativo e institucional, las mejoras en la infraestructura, el desarrollo curricular y la gestión académica. Metodológicamente se desarrolló un estudio de caso desde una perspectiva cualitativa en una institución de educación superior del sector no universitario en México. Como herramienta para recabar información se aplicaron entrevistas a actores clave para identificar coincidencias y discrepancias en su percepción acerca del impacto de la acreditación en su práctica cotidiana. Como principal hallazgo se observa que, desde la percepción de sus actores, la inclusión de organismos acreditadores ha transformado la práctica docente y administrativa en la institución analizada. Se destaca el uso de herramientas de planeación y gestión para sistematizar los procesos. Asimismo, la incorporación de indicadores incide directamente en la toma de decisiones. Como efectos no deseados se observa un vacío conceptual que evidencia que, operativamente, la calidad educativa es reducida al cumplimiento de indicadores y metas.
\end{abstract}

Palabras clave: Acreditación de programas, calidad educativa, sistemas de gestión de la calidad.

\section{ACCREDITATION OF QUALITY OF EDUCATION AND ITS IMPACT ON ACADEMIC MANAGEMENT: THE CASE OF A NON-UNIVERSITY INSTITUTION IN MEXICO}

\begin{abstract}
This article analyzes the perceptions about the impact of educational program accreditation in the institutional management. As lines of analysis, we use system and institutional quality, infrastructure improvement, curriculum development and academic administration. The methodology consists in a case study using the qualitative perspective. Relevant actors were interviewed in order to identify similarities and differences in perceptions about the impact of accreditation in their daily practice. Our main finding shows that the inclusion of external accreditation organizations has positively transformed teaching and administrative practice in the institution analyzed. It highlights the use of planning and management tools to systematize the educative processes. Also, indicators seem to have a direct effect on decision making processes. As a negative effect, quality is misunderstood just as the fulfillment of institutional goals and indicators.
\end{abstract}

Keywords: Program accreditation, education quality, quality management systems

1 Doctor en Ciencias Económico-Administrativas y maestro en Gestión y Políticas de la Educación Superior por la Universidad de Guadalajara. Universidad de Guadalajara, Centro Universitario de Ciencias Económico-Administrativas, Guadalajara, México.

Contacto: ivan.salas@cucea.udg.mx 


\section{LA ACREDITACIÓN DE LA CALIDAD EDUCATIVA Y LA PERCEPCIÓN DE SU IMPACTO EN LA GESTIÓN ACADÉMICA: EL CASO DE UNA INSTITUCIÓN DEL SECTOR NO UNIVERSITARIO EN MÉXICO}

\section{Introducción}

Derivado de la expansión de la oferta educativa del nivel superior suscitada en México durante las décadas de los ochenta y noventa, las instituciones han situado la calidad educativa dentro de su agenda. Debido a que es uno de los ejes fundamentales para el desarrollo, las instituciones de educación superior (IES) y el subsistema de educación superior han adoptado sistemas de gestión para medir y evaluar el impacto de sus programas de estudio (DeVries, Moheno y Romero, 2006). Como consecuencia de ello se han reconfigurado las actividades académicas y de gestión a un enfoque basado en la atención de necesidades específicas. Prueba de lo anterior es la inclusión de aspectos como la acreditación de organismos externos, la certificación de procesos administrativos, el establecimiento de convenios de colaboración con el sector social y productivo, así como la formación y capacitación integral de alumnos y profesores en los planes de desarrollo institucional (PDI).

Esto ha transformado la práctica docente y administrativa de las instituciones para incorporar elementos de planeación y gestión orientados a reducir la discrecionalidad en la toma de decisiones, gracias a la eficiencia y eficacia en la utilización de los recursos. Como resultado, al interior de la institución se sistematizan los procesos, en tanto que hacia el exterior, la organización valida ante la sociedad la calidad de sus servicios educativos. Sin embargo, no todos concuerdan con los beneficios que esto supone. Fernández y Gijón (2011) señalan que las políticas de aseguramiento de la calidad no son neutrales ni inocentes, sino que, por el contrario, poseen un sesgo que implícitamente parte de la base de que las IES no operan 
con eficiencia y es necesario supervisar la manera en la que utilizan sus recursos. Bernasconi (2008) profundiza afirmando que en las universidades públicas los cargos administrativos y directivos son ocupados por miembros de la comunidad universitaria. De manera que la falta de profesionalización en temas de calidad educativa conduce, inevitablemente, a una administración pragmática que imposibilita crear los contrapesos necesarios para neutralizar la dinámica reduccionista de los organismos acreditadores.

La pregunta de investigación que orienta al presente escrito es: ¿en qué medida ha modificado la gestión académica la inclusión de organismos externos que evalúan la calidad educativa? Para responder esta pregunta se tiene la hipótesis de que los organismos acreditadores han orientado la administración de las IES hacia una dinámica de sistematización de los procesos educativos a través de la planeación, seguimiento y evaluación de las actividades institucionales.

Para comprobar la hipótesis se llevó a cabo un trabajo de campo en una institución pública federal del sector no universitario que oferta cuatro carreras de ingeniería, de las cuales dos de ellas están acreditadas por organismos externos y las dos restantes no cuentan con esta distinción, porque son de reciente creación. Asimismo, dicha institución cuenta con un sistema certificado de gestión para la calidad que opera desde hace ocho años. Por lo tanto, posee las características asociadas a la lógica de la sistematización de los servicios educativos para hacer eficientes sus procesos y, de forma simultánea, validarse ante la sociedad.

\section{Marco referencial}

\subsection{Aproximación al concepto de calidad educativa}

Dias Sobrinho (2007) afirma que la atención hacia la calidad en la educación superior en Latinoamérica está originada en gran medida por la expansión y diversificación de la oferta educativa, así como los continuos cambios en la dinámica del sector productivo. Debido a la gran gama de IES, la calidad educativa es irregular. Esto hace necesario contar con parámetros generales que determinen el piso mínimo de 
operación en el sistema de educación superior. Sin embargo, Chávez (2006) advierte que no existen políticas integrales que pretendan establecer una metodología común aplicable a todos los estratos que ofertan educación superior, sino que se delega la acreditación de la calidad a organizaciones civiles sin la supervisión directa de la Secretaría de Educación Pública (Salas y Ordóñez, 2013). Por su parte, Cuevas (2011) profundiza en el tema al advertir que los organismos acreditadores son entidades con fines de lucro y, por consecuencia, son propensos a desviarse de sus objetivos.

Ahora bien, ¿cómo podemos definir la calidad educativa y, operativamente, cómo podemos constatarla? En la literatura se reportan diferentes enfoques y definiciones que nos permiten aproximarnos a la comprensión de este concepto.

La calidad en la educación es un concepto polivalente que depende del actor que utilicemos como referencia. Scharager y Aravena (2010) señalan que para unos implica empleabilidad, desarrollo de competencias o saberes; mientras que para otros se asocia al desarrollo humano y a las capacidades en ámbitos que van más allá del sector productivo. Paralelamente, en la literatura se aborda una vertiente complementaria que se enfoca en el análisis del proceso educativo. Implícitamente se parte de la idea de que un proceso controlado tendrá buenos resultados. Carr y Kemmis (1998) acotan la calidad al proceso constante de optimización del docente para desarrollar las capacidades formativas del estudiante. Casanova (2012) señala al respecto que la calidad se consigue a través del diseño curricular y potenciando la capacidad de sus profesores mediante la evaluación de cada una de las etapas que configuran la formación de los estudiantes. Se enfatiza en que no es un proceso genérico, sino que se requiere entender el contexto escolar y regional.

Murillo y Román (2010) hacen un llamado a asumir la complejidad e integralidad del concepto de calidad en la educación para evitar reducirla a unos cuantos parámetros, ya que desde su perspectiva se deben incluir otros factores tales como la integración entre niveles y la profesionalización de los directivos y del personal administrativo. 
González y Torre (2007) señalan que se puede evaluar mediante indicadores, criterios y variables que permitan garantizar a la sociedad la pertinencia de los saberes ofertados por las IES, los cuales se concentran tradicionalmente en rubros tales como los planes y programas de estudio, la plantilla docente, los alumnos, la infraestructura, el financiamiento y los procesos académico-administrativos (Gil, 2006). Esto significará, entonces, un mínimo de condiciones con las cuales deben operar las IES. El principal problema de esta manera de conceptualizar la calidad radica en las dificultades que acarrea su medición. En efecto, al existir un gran número de subsectores dentro del sistema de educación superior (por ejemplo, sector público y privado, de élite y de absorción, escolarizados, semiescolarizados y a distancia, universitario y no universitario) resulta prácticamente imposible amalgamar las diferentes visiones y objetivos para alinearlos dentro de un estándar establecido por una secretaría de gobierno que delega su revisión a particulares. Cuevas (2011) añade que en el sector privado la certificación de la calidad está condicionada por los recursos financieros de las IES creando un círculo de validaciónmarginación ${ }^{2}$.

Sin embargo, a nivel institucional la sistematización de procesos y la creación de indicadores establecen las bases para una mejor operación académica y administrativa. Por ejemplo, permiten establecer metas a corto, mediano y largo plazo, las cuales se evidencian en los PDI que desarrolla cada IES. Lemaitre (2009) señala que esta estandarización, en la mayoría de los casos, es sumamente conservadora, planteando escenarios ideales que terminan por dejar de lado la composición diversa del sistema de educación superior. Rodríguez, Fleet y Delgado (2009) añaden que la acreditación puede ser percibida como un mecanismo de autorregulación, aunque valdría la pena preguntarse si esa reflexión proviene de las propias instituciones o si, artificialmente, es implantada por organismos superiores. Al margen de lo anterior, los autores destacan que la información generada servirá para tomar mejores decisiones.

2 Esto significa que, quien cuenta con los recursos para acreditarse, se valida ante la sociedad por su calidad, correspondiendo típicamente a las instituciones de élite; mientras que las IES de absorción de la demanda suponen una dudosa calidad, ya que en principio no cuentan con las capacidades económicas para entrar en la dinámica de la acreditación de sus programas. 
Lo anterior hace suponer que no queda totalmente claro cuál es el propósito real del aseguramiento de la calidad, ya que puede ser concebido como un mecanismo de regulación de la oferta, motor para la mejora continua, o bien, como un elemento generador de información para la toma de decisiones (Zapata y Tejeda, 2009). Ante este vacío teórico es factible esperar que la acreditación de la calidad educativa sea una herramienta que permita sistematizar los procesos con la doble finalidad de, por una parte, buscar la eficiencia en el manejo de los recursos y, por otra, de contar con elementos para tomar mejores decisiones. Precisamente, si acotamos el alcance de la acreditación a estos elementos, podemos romper los imaginarios que se han construido alrededor de esta como la fórmula operativa para cuantificar la calidad educativa.

\subsection{La acreditación de la calidad en México}

Durante la década de los noventa fueron implementadas en México algunas políticas focalizadas en evaluar la calidad del sistema de educación superior desde sus procesos. Es entonces que se crean los Comités Interinstitucionales para la Evaluación de la Educación Superior (CIIES). Desde sus inicios su accionar se ha limitado a la evaluación diagnóstica de los programas educativos, sin que implique su acreditación. No fue hasta el año 2000 que la Secretaría de Educación Pública (SEP) reconoce al Consejo para la Acreditación de la Educación Superior (COPAES) y le delega a este la función de acreditar los programas académicos de educación superior en instituciones públicas y privadas (Buendía 2011). Cabe señalar que el COPAES es un organismo no gubernamental, sin fines de lucro, integrado exclusivamente por mexicanos. Estrictamente hablando, COPAES no acredita las carreras, sino que avala a los organismos que, de manera operativa, evalúan los programas académicos. Entre sus funciones se cuentan las siguientes:

a) Elaborar criterios y lineamientos para reconocer formalmente a los organismos acreditadores.

b) Formular un marco común de evaluación para la acreditación de la calidad.

c) Evaluar a los organismos acreditadores.

d) Hacer pública la relación de organismos acreditadores reconocidos. 
e) Supervisar el rigor académico e imparcialidad de los organismos acreditadores (COPAES, 2013).

En la actualidad operan en México 327 organismos acreditadores distribuidos en todas las áreas del conocimiento. Por su parte, el proceso de acreditación de programas educativos en el país consta de cinco etapas:

a) Solicitud de acreditación.

b) Cumplimiento de las condiciones del marco general para los procesos de acreditación de programas académicos.

c) Un autoestudio que permite a las instituciones conocer de manera general sus insumos, procesos y resultados y la medida en que contribuyen a los objetivos institucionales a partir de una guía ${ }^{4}$ proporcionada por el organismo acreditador.

d) La evaluación por parte de un organismo acreditador.

3 Asociación para la Acreditación y Certificación de Ciencias Sociales, A.C. (ACCECISO), Acreditadora Nacional de Programas de Arquitectura y Disciplinas del Espacio Habitable, A.C. (ANPADEH), Asociación Nacional de Profesionales del Mar, A.C. (ANPROMAR), Comité para la Acreditación de la Licenciatura en Biología, A.C. (CACEB), Consejo de Acreditación de la Enseñanza en la Contaduría y Administración, A.C. (CACECA), Consejo de Acreditación de la Enseñanza de la Ingeniería, A.C. (CACEI), Consejo para la Acreditación de la Educación Superior de las Artes, A.C. (CAESA), Comité para la Evaluación de Programas de Pedagogía y Educación, A.C. (CEPPE), Consejo Nacional para la Enseñanza e Investigación en Psicología, A.C. (CNEIP), Consejo para la Acreditación de Programas Educativos en Humanidades, A.C. (COAPEHUM), Consejo Mexicano para la Acreditación de la Enseñanza de la Cultura de la Actividad Física, A.C. (COMACAF), Consejo Mexicano de Acreditación y Certificación de la Enfermería, A.C. (COMACE), Consejo Mexicano para la Acreditación en Optometría, A.C. (COMACEO), Consejo Mexicano para la Acreditación de la Educación Farmacéutica, A.C. (COMAEF), Consejo Mexicano para la Acreditación de la Educación Médica, A.C. (COMAEM), Consejo Mexicano para la Acreditación de Programas de Diseño A.C. (COMAPROD), Comité Mexicano de Acreditación de la Educación Agronómica, A.C. (COMEAA), Consejo para la Acreditación de la Comunicación, A.C. (CONAC), Consejo Nacional para la Acreditación de la Ciencia Económica, A.C. (CONACE), Consejo Nacional de la Eseñanza y del Ejercicio Profesional de las Ciencias Químicas A.C. (CONAECQ), Consejo para la Acreditación de la Enseñanza del Derecho A.C. (CONAED), Consejo Nacional de Educación Odontológica, A.C. (CONAEDO), Consejo Nacional para la calidad de la Educación Turística A.C. (CONAET), Consejo Nacional de la Acreditación en Informática y Computación, A.C. (CONAIC), Consejo Nacional para la Calidad de los Programas Educativos en Nutriología, A.C. (CONCAPREN), Consejo Nacional de Educación de la Medicina Veterinaria y Zootecnia, A.C. (CONEVET), Consejo Nacional para la Acreditación de la Educación Superior en Derecho, A.C. (CONFEDE).

4 Se consideran once aspectos: filosofía institucional; planeación y efectividad; normatividad, gobierno y administración; programas académicos; personal académico; estudiantes; personal administrativo; apoyos académicos; recursos físicos; recursos financieros y educación a distancia. 
e. El dictamen que determina si el programa académico es acreditado o no.

Rama (2009) señala que la acreditación ha servido como un mecanismo de diferenciación para identificar a aquellas instituciones que cubren ciertos requerimientos de calidad. Dias Sobrinho (2012) hace una crítica al respecto señalando que la acreditación tiene como principal objetivo el control burocrático, legal y formal de la garantía de calidad.

Pires y Lemaitre (2008) afirman que la acreditación y las políticas de aseguramiento de la calidad generan información que permite a estudiantes, empleadores, instituciones y gobierno tomar mejores decisiones. Al mismo tiempo, permiten establecer puntos de comparación entre instituciones en términos de su desempeño. Dado su alcance, el gasto realizado en la implementación de sistemas de gestión de la calidad resulta marginal, ya que son un mecanismo efectivo para transparentar la información relativa al sistema educativo y como consecuencia se fortalece el sistema democrático y de participación ciudadana.

Lo que no resulta marginal es la transformación interna que deben sufrir las instituciones para estar en condiciones de implementar un sistema de gestión para la calidad. En este sentido es posible identificar cinco elementos relacionados entre sí para pensar en una adopción exitosa de este modelo. Dichos elementos se describen a continuación.

\section{a) Capacitación de la organización acerca de los conceptos asociados a la calidad educativa}

La adopción de un sistema para la gestión de la calidad no es el resultado únicamente de la elaboración de planes, programas, procedimientos e indicadores. Por el contrario, también requiere de la adopción de un marco teórico-conceptual que permita a los actores involucrados en los procesos entender las implicaciones de la calidad en la educación. Adicionalmente, Jiménez y López (2006) señalan que para implementar un sistema de gestión para la calidad a nivel organizacional se requiere de seis etapas: compromiso, planeación, 
desarrollo, implementación, registro y mejora. En este contexto la capacitación del personal no radica exclusivamente en temas asociados a la calidad, sino también a la planeación y a la gestión de recursos.

En la literatura es recurrente encontrar críticas a la adopción de sistemas de calidad que pretenden estandarizar la educación (Dias Sobrinho, 2012; Marúm, 2012; Rosario, 2012). El argumento central se basa en las particularidades de la educación, enfatizando en que no es un proceso fabril que consiste en «depositar» mecánicamente el conocimiento en los estudiantes. Sin embargo, si la adopción de un sistema de gestión para la calidad se concibe como una herramienta que permite a las instituciones y docentes contar con un marco de operación es posible pensar en un cambio organizacional. Para que esto pueda llevarse a cabo se requiere capacitar no solo a docentes, sino a toda la organización, de manera de asimilar el potencial de contar con procesos autorregulados. Del mismo modo, es necesario tener conceptos claros y sencillos que modifiquen el imaginario colectivo acerca de que la estandarización es un arma que atenta contra la libertad de cátedra.

\section{b) Adaptación de la estructura y organigrama}

El cambio organizacional no solo supone cambios en la actitud de los actores, sino también en la estructura y en las funciones que desempeñan. Así, si se pretende adoptar un sistema para la gestión de la calidad, se requiere de una revisión del organigrama y de las funciones de cada puesto. Si ello no ocurriera, se presentaría una incompatibilidad y duplicidad en las funciones, lo que derivará en prácticas de simulación. Estos cambios requieren de recursos e infraestructura que los soporten, por lo cual las instituciones tienen que reasignar su presupuesto para atender nuevas funciones y puestos.

\section{c) Combate de la resistencia al cambio}

Otro de los elementos clave para llevar a cabo cualquier cambio en las organizaciones es romper la inercia. Gairín y Rodríguez (2011) señalan que la innovación es la mejor forma de modificar la conciencia colectiva, la cual surge de la iniciativa de algunos agentes que detectan áreas de oportunidad en sus propios procesos y que cobran un carácter 
institucional cuando se encuentran alineados con los objetivos de las IES. Lo anterior implica que la innovación depende del contexto y de las características de sus agentes. En ese sentido, la implementación de un sistema para la gestión de la calidad requiere de líderes académicos que convenzan a sus pares de las bondades de un marco de operación homogéneo. De esta manera se generará la sinergia que permitirá establecer los mecanismos adecuados para el control de la calidad educativa, ya que no existe una fórmula única sino que son las instituciones, por intermedio de sus actores, las que determinarán los aspectos que desean abordar para controlar sus procesos.

\section{d) Replanteamiento de las funciones docentes}

Tradicionalmente, la labor docente es concebida dentro del aula y sus funciones se limitan a este espacio. Sin embargo, en la actualidad los profesores realizan una amplia gama de actividades en diferentes ámbitos: reforma y actualización curricular, vinculación con el sector social y productivo, administración de proyectos y recursos, elaboración de materiales de apoyo, capacitación continua, trabajo colegiado y participación en eventos institucionales.

Esta nueva dinámica exige que los profesores dediquen parte de su jornada laboral para llevar a cabo estas actividades. Es entonces que, con los sistemas de gestión para la calidad, se pueden replantear formalmente las funciones y atribuciones de la plantilla docente. Desde esta mirada se crearán las condiciones para elevar su presencia y rango de influencia a nivel institucional, democratizando su participación en la toma de decisiones. Esto nos remite al punto número primero, ya que se requiere de la existencia de claridad conceptual por parte de los actores para entender las implicaciones de esta nueva práctica docente.

\section{e) Sistemas robustos de gestión de información}

Finalmente, los cambios en la organización no operan en el vacío, sino que requieren de los recursos adecuados para poder ser implementados. La capacidad de procesamiento y flujo de la información abre la puerta para nuevas formas de gestión académica y administrativa. No es posible concebir un sistema eficiente y eficaz 
sin que se pueda acceder a través de la red a todos los planes, metas institucionales, reglamentos, procedimientos, formatos e indicadores.

Al margen del valor agregado que generan en la operación académica y administrativa, los sistemas de gestión de la información transparentan el desempeño de las instituciones, lo cual asigna la componente de rendición de cuentas ante la sociedad y permite llevar a cabo comparaciones entre instituciones con el objetivo de diferenciarlas de acuerdo con la calidad de sus servicios. Desde esta perspectiva, la acreditación externa crea las bases para transparentar el sistema de educación superior.

En relación con la implementación de sistemas para la gestión de la calidad, Cedeño y Hernández (2011) señalan que a nivel institucional los procesos de acreditación generan aprendizaje organizacional. Esto se evidencia en políticas institucionales, sistematización de los procesos de autoevaluación, implementación de acciones de mejora continua e identificación de perfiles docentes. De esta forma, en la medida en que los actores se involucren en este proceso, la organización se beneficiará directa e indirectamente, debido a que las funciones académicas tenderán a estandarizarse $y$, por consecuencia, se reducirá el margen de discrecionalidad de los individuos para sustituirlo por criterios racionales (Lemaitre, Maturana, Zenteno y Alvarado, 2012). Lo anterior no supone una automatización fabril de la educación, ya que las instituciones dotan a sus profesores de un marco de referencia a partir del cual basar su práctica, pero se respetan las singularidades de la relación maestroalumno, aportando elementos de planeación y administración de recursos que permiten enriquecer la formación de los estudiantes y aprovechar plenamente el potencial existente, tanto de la planta académica como de la infraestructura y servicios de la institución.

En cuanto a los efectos no deseados presentes en los procesos de acreditación educativa, Salas y Murillo (2008) señalan cinco aspectos:

- Falta de conceptos claros respecto de la calidad y la evaluación, ya que son medidas indirectamente por medio de indicadores, dejando de lado aspectos cualitativos fundamentales para evaluar la calidad de un programa académico. 
- Desvinculación entre la planeación y la evaluación, ya que los programas de desarrollo institucional y los lineamientos para la acreditación operan con distintas lógicas, lo cual incentiva la construcción de indicadores.

- Selección inadecuada de métodos y herramientas, debido a que las mediciones son indirectas. Adicionalmente, la revisión de indicadores es semejante a una auditoría en la que solo se comprueba la existencia de la evidencia, sin evaluar su calidad o pertinencia.

- Uso inadecuado de los resultados, ya que en el mejor de los casos sirven para dar validez externa a las IES, aunque no sean consideradas en los procesos de planeación, gestión, presupuestación, operación y autoevaluación.

- Ausencia de condiciones para llevar a cabo evaluaciones continuas, ya que la evaluación es percibida como un proceso único, realizado exclusivamente por expertos en el área y, en la mayoría de los casos, los resultados obtenidos no son socializados a la comunidad académica.

Murillo y Román (2010) complementan esta idea afirmando que la evaluación externa ha frenado el crecimiento del sistema educativo, dado que su lógica ha fragmentado y reducido los parámetros de calidad, orientando que los programas y sistemas educativos funcionen sobre la base de evaluaciones de matemáticas, lengua y ciencias.

\subsection{Caso de estudio: el Centro de Enseñanza Técnica Industrial (CETI)}

El CETI es una institución pública federal descentralizada de educación media superior y superior. Oferta carreras del área de las ingenierías y está ubicado en la zona metropolitana de Guadalajara en dos planteles, Colomos y Tonalá. El presente estudio se llevó a cabo en el plantel Colomos, dado que atiende el 91,3\% de la matrícula del nivel superior, es decir, aproximadamente a 2.100 estudiantes. Actualmente la institución oferta cuatro carreras: Ingeniería Mecatrónica, Ingeniería Industrial, Ingeniería en Diseño Electrónico y Sistemas Inteligentes e Ingeniería en Desarrollo de Software. 
Al ser una institución con vocación a la enseñanza tecnológica, tiene como principal objetivo el desarrollo de capacidades conceptuales y técnicas para la generación y aplicación de conocimiento enfocado en la solución de problemas. Complementariamente, en la misión se hace referencia a la prestación de servicios tecnológicos en áreas estratégicas y en el fomento de la investigación que coopere con el desarrollo de la región a la que pertenecen.

En términos de la calidad educativa, cuenta con un sistema de gestión acreditado por la Agencia para la Certificación de la Calidad y el Medio Ambiente (ACCM) implementado hace ocho años que, junto con la normativa, rige los procesos de administración académica. Las carreras de Ingeniería Mecatrónica e Ingeniería Industrial cuentan con la acreditación de sus programas educativos por parte del Consejo de Acreditación de la Enseñanza de la Ingeniería A.C. (CACEI). Mientras que las dos carreras restantes todavía no son candidatas a este tipo de reconocimiento, dado que son de reciente creación. Cabe destacar que, derivado de un proceso de reforma curricular, se replantea la carrera de Ingeniería Electrónica para transformarla en los dos programas señalados. Como referencia, esta cuenta con acreditación vigente por parte del CACEI, de manera que la plataforma de las nuevas carreras está basada en los requerimientos de los organismos evaluadores. Operativamente, los coordinadores de las carreras de reciente creación cuentan con la experiencia de haber participado en la acreditación de la carrera de Ingeniería Electrónica, por lo que a pesar de no contar con este reconocimiento, están inmersos en la lógica de los organismos acreditadores. Prueba de ello es su nivel de conocimiento del tema, ya que en las entrevistas no se encontraron diferencias significativas en sus respuestas respecto de las de los coordinadores de carreras acreditadas.

\section{Metodología}

El estudio de caso del cual habla el artículo utiliza una metodología cualitativa. Se analiza la percepción de los coordinadores de las carreras respecto del impacto que ha tenido la acreditación de los programas educativos en su práctica cotidiana. Para ello, se abordan los siguientes ejes: el panorama actual de la calidad del sistema 
educativo, la calidad educativa a nivel institucional, mejoras en la infraestructura, desarrollo curricular y gestión académica. Cabe señalar que dichos ejes, así como la entrevista, corresponden a una adaptación de la metodología planteada por el Laboratorio de Análisis Institucional del Sistema Universitario Mexicano para un estudio desarrollado para CINDA y cuyo objetivo es evaluar el impacto de la acreditación en seis universidades mexicanas a partir de la información provista por actores relevantes ${ }^{5}$ (Buendía, 2011).

Respecto de los informantes clave se seleccionaron a los coordinadores de las carreras dado que sus funciones integran tanto la administración académica, la docencia, la atención a estudiantes y el diseño curricular, como la gestión de recursos e infraestructura, además de ser participantes activos en la acreditación de sus carreras. Por ello su grado de inmersión en el tema de la calidad educativa es alto, ya que formalmente operan los procesos de administración académica y de enseñanza-aprendizaje.

Como herramienta para la obtención de información se adaptó el cuestionario planteado por Buendía (2011) para llevar a cabo una entrevista en profundidad a los informantes, respetando los ejes analíticos que plantea. En la gráfica 1 se muestran las categorías empleadas para el análisis.

5 Cabe señalar que el proyecto original planteado por CINDA realizó un estudio en toda Latinoamérica para evaluar el impacto de la acreditación en la calidad educativa, por lo que es posible acceder a los informes de cada uno de los países que participaron en dicha investigación. 
Gráfica 1: Ejes de análisis.

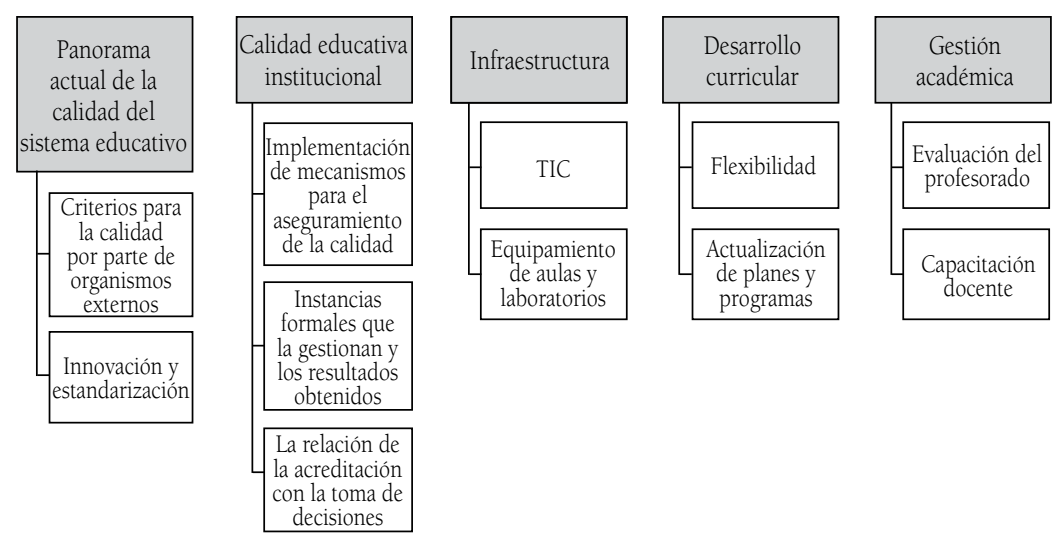

Fuente: Elaboración propia.

En total se aplicaron cinco entrevistas en profundidad durante el mes de octubre y noviembre de 2012. Cuatro de ellas corresponden a los coordinadores de las carreras, en tanto que la quinta se realizó al coordinador del área de tronco común, el cual se encarga de la administración de las academias de física y química, matemáticas, ciencias sociales y lengua extranjera, las cuales son comunes a todas las carreras. De esta manera se abarcó el universo de coordinadores de carrera.

\section{Resultados}

Respecto de la percepción acerca de la gestión de la calidad del sistema educativo se encontraron opiniones encontradas. Por un lado, se asume que los organismos acreditadores mexicanos basan sus criterios en normas y estándares internacionales, dando como resultado el prestigio a las instituciones que son acreditadas; y, por otra parte, se hace mención a que no existe un organismo único con la facultad de coordinar a las casas acreditadoras bajo un esquema común. Asimismo, no pareciera existir un sustento estadístico que avale los criterios que cuantifican la calidad educativa. En ese sentido, se señala que tales criterios se basan tanto en la normativa como en la propia experiencia de las casas acreditadoras para generar la guía de autoevaluación. Esta asimetría se debe, en gran medida, a que se pone en acción una política gubernamental mediante organizaciones civiles, 
lo cual trae como consecuencia un vacío conceptual que dificulta entender qué debemos esperar de las instituciones en términos de su calidad. Si a lo anterior añadimos que el sistema de educación superior está compuesto por diferentes estratos, no resulta extraño que no exista una idea clara acerca de los objetivos reales que tienen las casas acreditadoras, creándose entonces un imaginario institucional que asigna calidad moral a las observaciones planteadas a los programas.

Por otra parte, asociado al eje de análisis del sistema educativo está el tema de la innovación y la estandarización de los procesos. Los entrevistados coinciden en que es necesario que exista un piso mínimo a partir del cual deben operar las IES y en su percepción no limita la innovación. Del mismo modo, se hace referencia a que las instituciones imprimen su sello distintivo en aspectos tales como las asignaturas de las carreras, la atención de las necesidades específicas del sector productivo regional, las políticas y los objetivos particulares de acuerdo con su vocación, al margen de los criterios de los organismos acreditadores. Esto afirma la idea de que los actores involucrados en los procesos de administración académica están convencidos de que su labor es capaz de establecer mejoras en los procesos. Desde esta mirada, la acreditación de las carreras es el mínimo necesario de calidad y serían las propias instituciones las que, con sus actores, establecen el alcance de sus programas. En términos de un informante:

Es imposible poder ser competitivo sin un grado de homogeneidad en estos tiempos, dado que los requisitos de los usuarios finales (industria, etc.) son bastante claros y son los mismos para toda universidad. Lo anterior no debe ser una limitante para la innovación, pues si un estudio de pertinencia avala las modificaciones, estará a su vez acorde con las expectativas de los usuarios finales, por ende, cerrando el círculo. Coordinador 2.

Es factible pensar que la acreditación de la calidad per se no genera ni limita la innovación institucional, sino que concientiza e incentiva a los individuos a repensar las prácticas institucionales. En la medida en que esto se masifica es posible consolidar una cultura de la calidad que impacte en los procesos educativos. Sin embargo, también se corre el riesgo de definir, sistematizar, medir y evaluar 
los procesos para que nada cambie. En otras palabras, la adopción de este tipo de sistemas permite realizar las mismas actividades, con sus limitaciones y potencialidades, con la única diferencia de que se deja una evidencia de su ejecución. En ese sentido, no se verifica en el testimonio de los actores entrevistados una relación directa que permita asociar la estandarización con la innovación en los procesos académicos o administrativos.

En cuanto al eje institucional de la calidad educativa y su relación con la acreditación de los programas, se abordaron los siguientes temas: la implementación de mecanismos para el aseguramiento de la calidad, las instancias formales que la gestionan, los resultados obtenidos y su relación con la toma de decisiones y las estrategias institucionales que impactan directamente a los estudiantes.

Respecto de los sistemas de gestión de la calidad, los entrevistados coinciden en que durante los últimos cinco años se ha observado una transformación de las funciones académicas y administrativas para operar los programas. Se hace referencia al sistema de gestión de calidad (SGC) implementado en la institución, el cual ha tenido como resultado la definición de procedimientos. De esta manera, los coordinadores de las carreras tienen la certeza de los indicadores en torno a los cuales se sustentan sus acciones. Asimismo, la administración de los docentes se lleva a cabo mediante una carpeta académica que reúne información del proceso de enseñanza-aprendizaje. Sin embargo, uno de los coordinadores hace mención de que el proceso de planeación y la generación de indicadores se lleva a cabo sin que existan etapas intermedias de evaluación que permitan tomar acciones preventivas. De igual manera, existe resistencia al cambio por parte de los profesores, ya que no todos han adoptado por completo la forma de trabajo que supone una estandarización. Como resultado de lo anterior se han consolidado algunos procesos, mientras que en otros se presentan prácticas de simulación. Ante esto, cobra sentido la capacitación del personal en aspectos asociados a la calidad educativa, ya que si las acciones no cuentan con un objetivo claro, tenderán a disiparse y a perder impacto. Esto nos lleva a pensar que la dimensión normativa es solo la mitad de la ecuación, ya que corresponde a los actores que el «deber ser» se aplique en la práctica cotidiana. 
Las instancias encargadas de la gestión de la calidad mencionadas por los informantes son varias, ya que se señalan cinco departamentos que están involucrados en este proceso. Esto se puede interpretar en el sentido de que la calidad educativa relaciona a todas las partes de la organización. Así mismo, implica que los resultados obtenidos dependen en buena medida de la coordinación de los agentes y de la manera en la que está alineada su labor con las metas institucionales. Esto nos lleva al personal que opera los procesos, ya que el éxito de los sistemas de gestión de la calidad no depende exclusivamente de la definición de funciones y de los procesos, sino de la madurez organizacional de su personal operativo para asimilar y llevar a la práctica el concepto de calidad en la educación. En esta línea, los entrevistados no hacen mención a un ejemplo exitoso de articulación entre áreas, lo cual haría suponer que en el discurso esto se lleva a cabo, aunque en la realidad las áreas operan de manera independiente.

Es importante señalar que los resultados obtenidos son utilizados para la toma de decisiones. Se destaca la creación de nuevas carreras o la adecuación de los programas de estudio, atendiendo a las observaciones de los organismos acreditadores y a las necesidades del sector productivo. De igual manera, derivado de las observaciones presentadas por las partes involucradas, se han implementado mejoras al sistema de control escolar, así como también estrategias institucionales para disminuir la deserción y la reprobación estudiantil.

Es factible afirmar que la definición y estandarización de los procesos, junto con la retroalimentación de las partes involucradas, llevan a la mejora continua de los procesos educativos. Esto se puede evidenciar en los planes de trabajo anual que realizan los coordinadores de carrera, los cuales están alineados al PDI, lo que supone un equilibrio dinámico entre las necesidades externas y la lógica interna.

Finalmente, la calidad educativa forzosamente deberá ser observada por los estudiantes mediante programas y acciones focalizadas en auxiliarlos en su tránsito por la institución. Los 
entrevistados señalan como los principales elementos de este eje las asesorías extraacadémicas que se imparten a los alumnos con algún nivel de retraso, la atención psicopedagógica por el Gabinete de Orientación Educativa (GOE) y el seguimiento a los estudiantes desertores para entender las causas por las cuales los jóvenes abandonan sus estudios; todo ello con el fin de establecer estrategias de contención. Igualmente, la evaluación de los docentes y los mecanismos implementados para la selección y contratación de estos contribuyen a mejorar la atención de los estudiantes. En palabras de un coordinador:

Los resultados de la evaluación nos permiten tomar medidas y acciones pertinentes en la mejora continua del propio docente. Se retroalimenta la información con cada uno de los docentes y se le hace ver sus fortalezas y sus debilidades para que trabaje en ellas con una actitud al cambio y al servicio del alumnado, para el bien propio y de los demás. En algunas ocasiones nos ha permitido no recontratar al docente por su falta de profesionalismo o entrega a su vocación de servicio. Coordinador 3.

En ese sentido, la acreditación de la calidad educativa ha tenido el efecto de regular en mayor o menor grado la práctica docente, ya que existe un registro histórico de las evaluaciones a estos. Esta información es utilizada para la toma de decisiones, al menos en el personal de contrato temporal, orientando el desempeño de los docentes hacia las necesidades institucionales. Complementariamente, el personal de contrato definitivo no se sujeta a esta lógica, ya que su permanencia en la institución no está ligada a su desempeño docente. Esto evidencia que la evaluación de la calidad educativa es aplicada asimétricamente, puesto que el nivel de involucramiento del personal depende de su situación contractual, lo que supone prácticas ocultas que se disimulan en los indicadores.

En lo que respecta al eje analítico de la infraestructura, se reconoce que durante los últimos cinco años han existido esfuerzos importantes para mejorar las condiciones en las que se imparten las carreras. Se destaca la creación de un edificio planteado para ampliar la matrícula del nivel superior. También se hace referencia a la compra 
de equipo especializado de laboratorio y material multimedia como proyectores, computadores y aulas interactivas para apoyar a los docentes y existe una percepción positiva acerca del acceso a los sistemas de información. Recurrentemente se señala la apertura y transparencia en la que se puede capturar y consultar información del proceso de enseñanza-aprendizaje. En ese sentido, el fortalecimiento de la infraestructura es un elemento deseable que permite elevar la calidad educativa; sin embargo, se requiere de capacitación para que el personal explote el potencial de las TIC, para que sean herramientas realmente efectivas.

Cabe señalar que el CETI ha participado en fondos extraordinarios para captar recursos para equipar sus laboratorios y la institución lleva tres años en el Programa de Apoyo al Desarrollo de la Educación Superior (PADES). De esta manera se han atendido los requerimientos planteados por el CACEI en términos de equipamiento para la realización de prácticas. A pesar de este esfuerzo, los fondos extraordinarios han sido marginales e insuficientes para atender las necesidades de los estudiantes ${ }^{6}$, ya que se menciona una desactualización histórica en el equipamiento de los laboratorios.

Respecto del eje de desarrollo curricular, los informantes apuntan a la flexibilidad curricular como el principal logro de la mejora de la calidad educativa. Literalmente:

El cambio de sistema rígido al sistema por créditos, donde existe mayor flexibilidad para cursar la carrera. Mínimo tres años a un máximo de seis años, por lo que las carreras se adaptan a la disponibilidad del alumno; y él toma las materias de acuerdo con sus necesidades. Coordinador 1 .

El cambio en el modelo educativo es el resultado de un estudio de pertinencia para adaptarse a las necesidades de los usuarios.

6 Para dimensionar el peso de los fondos extraordinarios consideremos que en los últimos tres años se han obtenido aproximadamente tres millones de pesos. Esto contrasta con el presupuesto anual del CETI, el cual es de aproximadamente ciento veinte millones de pesos. Esto significa que menos del $1 \%$ de los recursos provienen de fondos especializados en fortalecer la infraestructura y equipo de laboratorio. 
En ese sentido, se atienen directamente a las recomendaciones de los organismos acreditadores en relación con la flexibilización de la oferta educativa. De igual manera, se actualizan los programas teniendo como consecuencia la adaptación de los perfiles docentes, lo cual habla del esfuerzo institucional por atender las necesidades del sector productivo.

Para concluir, en cuanto al eje de la gestión académica los coordinadores de carrera hicieron referencia a los diferentes ámbitos en los que ha incidido el tema de la calidad educativa. En cuanto a la selección y contratación de docentes se mencionan procesos específicos que evitan la discrecionalidad, aplicando exámenes y grados académicos como elementos para la toma de decisiones. Lo anterior indica que, implícitamente, el SGC ha transparentado algunos mecanismos institucionales. De esta forma se abre la puerta a un sistema más democrático e incluyente. Se puede inferir también que, previamente a la implementación del sistema de gestión, la contratación no estaba normada y era susceptible a la voluntad de los directivos. En ese sentido se puede identificar que la sistematización en la contratación conduce a esquemas de mérito en donde son empleados los mejores candidatos.

Por otra parte, la gestión de los docentes se realiza a través de una carpeta académica. Además del componente individual, lo anterior permite a los operadores de los programas recabar información que les facilite tomar decisiones racionales en cuanto al desarrollo de los cursos. Se emplean en este portafolio de evidencias herramientas de planeación, creación de indicadores y retroalimentación que cooperan para que las asignaturas cuenten con elementos comunes, independientemente del estilo de cada docente. Al respecto se señala:

Como docente nuestro compromiso es evaluar nuestro propio trabajo y cómo lo llevamos a cabo dentro y fuera del aula y entregar las evidencias necesarias del control y seguimiento del trabajo realizado, así como los desempeños de aprendizajes esperados del alumno.

La evaluación y la retroalimentación de los procesos de la gestión escolar es la clave fundamental para 
que las instituciones lleven un buen control de sus procedimientos marcados en el manual de la calidad.

El saber y hacer cumplir las normas y procedimientos dan la confianza de obtener una acreditación de carrera.

Retroalimentar (envío de información por correo electrónico, reuniones de academia, entre otras) que todo el personal adscrito al departamento conozca sus funciones, obligaciones, derechos, así como cumplir con las entregas en las fechas señaladas y en los formatos oficiales. Coordinador 3.

Sin embargo, el testimonio de los actores entrevistados hace énfasis en el compromiso y responsabilidad de los docentes, sin dejar claro si en la realidad todos cumplen con esta encomienda, o bien, cuáles son los mecanismos para asegurar que todos están al tanto de esta dinámica.

Otra tendencia que se observa es cómo las TIC han venido a apuntalar el trabajo de los docentes para actualizar sus metodologías de enseñanza. Por ejemplo, ello ha permitido que el uso de aulas interactivas, software de simulación especializado y herramientas de apoyo en línea impacten directamente en la calidad de la formación de los estudiantes. Se podría suponer que la formación del alumnado es congruente con las necesidades del sector productivo de la región. Prueba de ello son los comentarios vertidos por los empleadores en los estudios de pertinencia sobre los que se fundamentan las carreras. Una vez más se atienden las observaciones de los organismos acreditadores, no solo en un sentido estricto de cumplimiento, sino también en un espíritu de mejora continua.

El último aspecto señalado se refiere a la evaluación del docente. A raíz de la implementación del SGC y de la definición de procedimientos orientados a la mejora se creó un instrumento denominado «evaluación integral del docente». En él se pueden observar los elementos asociados al aseguramiento de la calidad. Por ejemplo, el instrumento incluye una autoevaluación, así como la percepción del coordinador de la carrera, sus pares académicos y de los estudiantes. También se hace mención al elemento de control, 
en este caso, los compromisos que hace el docente para mejorar su práctica. Esto nos lleva a retomar la diferenciación en el trato a los profesores a partir de su situación contractual, haciendo evidente que la evaluación es un requisito para algunos y la clave para una futura contratación para otros. Por último, si la evaluación de la calidad es irregular, los procesos de acreditación conllevan, implícitamente, un componente de simulación organizacional.

\section{Conclusiones}

A partir de la evidencia presentada se puede concluir que en la institución analizada existe un nivel consolidado de cultura de gestión de la calidad. Al ser los coordinadores de las carreras los encargados de supervisar buena parte de los elementos asociados al proceso de enseñanza-aprendizaje es factible pensar que su práctica tiene elementos claramente identificables de planeación, control y evaluación. Inclusive en el lenguaje en el que se respondió la entrevista se muestran conceptos comunes que resaltan una cultura institucional en la que están claros tanto los objetivos como las metas.

Se destaca también la inclusión de herramientas de planeación y gestión que han permitido sistematizar los procesos, lo cual ha conducido a la elaboración de indicadores que inciden directamente en la toma de decisiones. Son varios los ámbitos que abarca esta forma de trabajo, entre los cuales podemos mencionar: la selección y contratación de personal, la planeación, desarrollo y evaluación de los cursos, el equipamiento escalonado de laboratorios, la evaluación de los docentes, los procesos para la reforma y creación de programas de estudio, así como la gestión de las carreras. En ese sentido, las recomendaciones de los organismos acreditadores han servido como catalizador para establecer algunos lineamientos de operación de la institución y, a la vez, asignar un valor simbólico ante la sociedad.

La consolidación de un sistema de gestión para la calidad depende tanto del marco normativo como de la capacidad y disposición de los agentes involucrados. Gracias a ambos factores se podrá transformar el imaginario de la homogeneización de la calidad educativa como algo negativo, para convertirlo en una visión 
de eficiencia y eficacia de los procesos que permita, a docentes y administrativos, explotar todo su potencial a favor de las instituciones.

Como se observa, la implementación de un sistema de gestión para la calidad, en concordancia con las observaciones de organismos acreditadores, ha creado un conjunto de condiciones que han propiciado una mejora en la práctica docente y administrativa de la institución analizada. Es importante señalar que estos cambios han tenido un efecto positivo debido a la naturaleza de la institución, ya que al ser pública del sector no universitario se ha contrarrestado la resistencia al cambio en buena medida gracias a su normativa y estructura. Como asignatura pendiente se requiere replicar este estudio hacia otras IES de diferente naturaleza, para comparar el impacto que ha tenido la implementación de sistemas para la gestión de la calidad en la gestión académica.

Sin embargo, también pudo ser constatado que la inclusión de organismos acreditadores ha introducido una concepción pragmática de la calidad educativa, lo cual genera un vacío conceptual que conduce a que los actores reduzcan la calidad al cumplimiento de metas e indicadores institucionales. Esto puede ser constatado en el lenguaje común de los agentes entrevistados, el que si bien establece un marco de referencia, no «aterriza» la calidad o mejora la formación de los estudiantes para que, una vez que hayan egresado, puedan insertarse exitosamente en el mercado laboral; sino que se queda en el discurso de definir, registrar, controlar y medir los procesos existentes sin que ello implique necesariamente una mejora.

En un término más general, la acreditación de la calidad por organismos externos debería ser entendida como un conjunto de herramientas de gestión que, en paralelo a las políticas institucionales para el aseguramiento de la calidad, permita generar información confiable acerca de los procesos académicos que derive en la toma de mejores decisiones, lo cual es distinto de tratar de reducir la calidad educativa a indicadores, objetivos y metas. 


\section{Referencias}

Bernasconi, A., (2008) La profesionalización de la academia en Chile. Revista Calidad en la Educación, 28, pp. 15-27.

Buendía, A., (2011) Aseguramiento de la calidad: políticas públicas y gestión universitaria. Estudio sobre impacto de los procesos de aseguramiento de la calidad (AD) a partir de información provista por actores relevantes: el caso de seis universidades en México. México D.F.: Laboratorio de Análisis Institucional del Sistema Universitario Mexicano.

Carr, W., y S. Kemmis, (1998) Teoría crítica de la enseñanza. Barcelona: Martínez Roca.

Casanova, M., (2012) El diseño curricular como factor de calidad educativa. Revista Iberoamericana sobre calidad, eficacia y cambio en educación, 10(4), pp. 7-20.

Cedeño, M., y C. Hernández, (2011) Autoevaluación y acreditación de carreras en la Universidad Nacional de Costa Rica: experiencias para la gestión de la calidad. Revista Calidad en la Educación Superior, 2(2), pp. 44-68.

Chávez, M., (2006) Los procesos de evaluación y acreditación en México: reflexiones sobre experiencias en evaluación y acreditación de tres programas académicos de licenciatura en trabajo social. En V. Rosario; E. Marúm; R. Vargas; J. Arroyo, y V. González (coords.) Acreditación y certificación de la educación superior: experiencias, realidades y retos para las IES (pp. 149-160). México: Universidad de Guadalajara.

COPAES (2013) Estatutos COPAES. Disponible en http://www.copaes.org. $\mathrm{mx} /$ FINAL/docs/EstatutosCopaes.pdf

Cuevas, Y., (2011) Organismos y procesos de certificación de la calidad de la educación superior privada en México. Revista Calidad en la Educación, 35, pp. 231-254.

DeVries, W.; Moheno, M., y J. Romero, (2006) La acreditación en México: algunos avances con crecientes contradicciones. En V. Rosario; E. Marúm; R. Vargas; J. Arroyo y V. González (coords.). Acreditación y certificación de la educación superior: experiencias, realidades y retos para las IES (pp. 431-446). México: Universidad de Guadalajara.

Dias Sobrinho, J., (2012) Acreditación en América Latina: crisis de la educación superior y distintas fases de la calidad. En V. Rosario; A. Didriksson; E. Marúm; J. Dias Sobrinho; N. Fernández; F. López; E. Villanueva, y J. Ríos (coords). La acreditación de la educación superior en Iberoamérica. La gestión de la calidad de los programas educativos. Tensiones, desencuentros, conflictos y resultados. Volumen 1 (pp. 17-35). Bloomington: Palibrio. 
Dias Sobrinho, J., (2007) Acreditación de la educación superior en América Latina y el Caribe. En C. Sanyal, y J. Tres (coords.). La educación superior en el mundo 2007: Acreditación para la garantía de la calidad: ¿Qué está en juego? (pp. 282-295). Madrid: Mundiprensa.

Fernández, M., y J. Gijón, (2011) Nuevas políticas de profesionalización docente en la educación superior. Journal for Educators, Teachers and Trainers, 2, pp. 92-106.

Gairín, J., y D. Rodríguez, (2011) Cambio y mejora en las organizaciones educativas. Educar, 47(1), pp. 31-50.

Gil, H., (2006) La evaluación, acreditación en las instituciones de educación superior públicas de México: estrategias de calidad educativa. En V. Rosario; E. Marúm; R. Vargas; J. Arroyo y V. González (coords.). Acreditación y certificación de la educación superior: experiencias, realidades y retos para las IES (pp. 423-430). México: Universidad de Guadalajara.

González, L., y D. Torre, (2007) Acreditación y fomento de la calidad. La experiencia chilena de las últimas décadas. En C. Sanyal, y J. Tres (coords.). La educación superior en el mundo 2007: Acreditación para la garantía de la calidad: ¿Qué está en juego? (pp. 298-306). Madrid: Mundiprensa.

Jiménez, R., y M. López, (2006) Una reflexión sobre la certificación de los servicios de información del Centro Universitario de Ciencias Exactas e Ingeniería: contexto nacional y pertinencia institucional. En V. Rosario; E. Marúm; R. Vargas; J. Arroyo y V. González (coords.). Acreditación y certificación de la educación superior: experiencias, realidades y retos para las IES (pp. 77-86). México: Universidad de Guadalajara.

Lemaitre, M., (2009) Nuevos enfoques sobre aseguramiento de la calidad en un contexto de cambios. Revista Calidad en la Educación, 31, pp. 170-189.

Lemaitre, M.; Maturana, M.; Zenteno, M., y A. Alvarado, (2012) Cambios en la gestión institucional en universidades, a partir de la implementación del sistema nacional de aseguramiento de la calidad: la experiencia chilena. Revista Calidad en la Educación, 36, pp. 22-52.

Marúm, E., (2012) Actores de la calidad de la educación superior en América Latina y España: acuerdos y desacuerdos. En V. Rosario; A. Didriksson; E. Marúm; J. Dias Sobrinho; N. Fernández; F. López; E. Villanueva y J. Ríos (coords). La acreditación de la educación superior en Iberoamérica. La gestión de la calidad de los programas educativos. Tensiones, desencuentros, conflictos y resultados. Volumen 1 (pp. 36-57). Bloomington: Palibrio. 
Murillo, F., y M. Román, (2010) Retos en la evaluación de la calidad de la educación en América Latina. Revista Iberoamericana de Educación, 53, pp. 97-120.

Pires, S., y M. Lemaitre, (2008) Sistemas de acreditación y evaluación de la educación superior en América latina y el caribe. En A. Gazzola, y A. Didriksson (editores). Tendencias de la Educación Superior en América Latina y el Caribe. Caracas: IESALC-UNESCO.

Rama, C., (2009) El nacimiento de la acreditación internacional. Revista da Avaliação da Educação Superior, 14(2), pp. 291-311.

Rodríguez, E.; Fleet, N., y M. Delgado, (2009) La acreditación en la generación de información sobre la calidad de la educación superior. Revista Calidad en la Educación, 31, pp. 212-230.

Rosario, V., (2012) Repensar los procesos de acreditación en la universidad pública mexicana en el marco de su responsabilidad social. En V. Rosario; A. Didriksson; E. Marúm; J. Dias Sobrinho; N. Fernández; F. López; E. Villanueva y J. Ríos (coords). La acreditación de la educación superior en Iberoamérica. La gestión de la calidad de los programas educativos. Tensiones, desencuentros, conflictos y resultados. Volumen 1 (pp. 195-209). Bloomington: Palibrio.

Salas, I., y F. Ordóñez, (2013) La acreditación desde la perspectiva de sus actores: el caso del Centro Universitario de Ciencias Exactas e Ingenierías de la Universidad de Guadalajara. En V. Rosario; A. Didriksson; E. Marúm; J. Dias Sobrinho; N. Fernández; F. López; E. Villanueva y J. Ríos (coords). La acreditación de la educación superior en Iberoamérica. La gestión de la calidad de los programas educativos. Tensiones, desencuentros, conflictos y resultados. Volumen 2 (pp. 237-248). Bloomington: Palibrio.

Salas, I., y F. Murillo, (2008) Organismos acreditadores de la educación superior en México: reflexiones sobre su problemática. Memorias académicas del Congreso Internacional de Evaluación Educativa. Tlaxcala: Universidad Autónoma de Tlaxcala.

Scharager, J., y M. Aravena, (2010) Impacto de las políticas de aseguramiento de la calidad en programas de educación superior: un estudio exploratorio. Revista Calidad en la Educación, 32, pp. 16-42.

Zapata, G., y I. Tejeda, (2009) Impactos del aseguramiento de la calidad y acreditación de la educación superior. Consideraciones y proposiciones. Revista Calidad en la Educación, 31, pp. 192-209. 


\section{Anexo 1}

Guía de preguntas aplicadas a los agentes clave

1. ¿Cómo evalúa los procedimientos que llevan a cabo los organismos acreditadores para medir la calidad educativa?

2. ¿Qué mecanismos de aseguramiento de la calidad se han implementado al interior de su carrera y qué obstáculos ha observado para su operación?

3. ¿Existe alguna instancia técnica que administre el aseguramiento de la calidad de su carrera? Mencione el tipo de apoyo que brindan.

4. ¿Qué opina respecto del debate que supone la homogeneización de la educación en aspectos tales como la innovación y la diferenciación entre instituciones?

5. ¿En qué ámbitos concretos de la gestión académica se han observado cambios a raíz de la acreditación de las carreras?

6. ¿Cómo han evolucionado los sistemas de gestión de la información a raíz de la acreditación?

7. ¿En qué grado influye la información generada para la toma de decisiones?

8. ¿Durante los últimos cinco años la unidad académica a la que pertenece ha modificado sus objetivos, metas y/o indicadores?

9. ¿Existen sistemas de seguimiento para evaluar el cumplimiento de objetivos y metas institucionales?

10. Derivado de la acreditación, ¿qué cambios han sufrido los servicios administrativos?

11. Mencione los cambios que se han presentado en los últimos cinco años en términos de la gestión de los docentes.

12. Mencione los cambios que se han presentado en los últimos cinco años en términos de infraestructura, TIC y recursos bibliográficos.

13. ¿Han existido innovaciones para el aprendizaje y cuál ha sido su relación con la acreditación?

14. ¿Cómo se gestiona al cuerpo docente en su institución?

15. ¿De qué manera ha influido la acreditación en dichos cambios?

16. ¿Existen mecanismos para que participen grupos internos y externos en la evaluación de los programas académicos? 
17. ¿En qué medida influyen dichos mecanismos para la toma de decisiones?

18. Durante los últimos cinco años ¿qué cambios ha observado en cuanto a los siguientes elementos?:

- Tratamiento de alumnos en riesgo.

- Flexibilidad curricular.

- Articulación con el nivel medio superior.

- Definición y revisión de los perfiles de ingreso y egreso.

19. Mencione los esfuerzos institucionales para retener a los estudiantes y su relación con la acreditación de los programas.

20. ¿Cuáles han sido las innovaciones pedagógicas que se han presentado en su carrera en los últimos cinco años?

Recibido: 5/03/2013

Aceptado: 9/05/2013 